\title{
Autosomal dominant Brody disease cosegregates with a chromosomal $(2 ; 7)($ p11.2; 12.1$)$ translocation in an Italian family
}

\author{
Antonio Novelli ${ }^{1}$, Enza Maria Valente ${ }^{*, 1}$, Laura Bernardini ${ }^{1,2}$, Caterina Ceccarini ${ }^{1,2}$, \\ Lorenzo Sinibaldi $^{1,2}$, Viviana Caputo ${ }^{1,2}$, Pietro Cavalli ${ }^{3}$ and Bruno Dallapiccola ${ }^{1,2}$
}

\begin{abstract}
${ }^{1}$ IRCCS CSS, San Giovanni Rotondo and CSS Mendel, Rome, Italy; ${ }^{2}$ Dipartimento di Medicina Sperimentale e Patologia, Università La Sapienza, Rome, Italy; ${ }^{3}$ Servizio di Genetica, Azienda Istituti Ospitalieri di Cremona, Cremona, Italy
\end{abstract}

Brody disease is a rare muscle disorder characterized by exercise-induced impairment in muscle relaxation, due to a markedly reduced influx of calcium ions in the sarcoplasmic reticulum. A subset of autosomal recessive families harbour mutations in the ATP2A1 gene, encoding the fast-twitch skeletal muscle sarcoplasmic reticulum $\mathrm{Ca}^{2+}$ ATPase (SERCA1). Rare autosomal dominant families have been described, in which ATP2A1 was excluded as the causative gene, further supporting genetic heterogeneity. We report four individuals from a three-generation Italian family with a clinical phenotype of Brody disease, in which linkage analysis excluded ATP2A1 as the responsible gene. The disease cosegregates in an autosomal dominant fashion with an apparently balanced constitutional chromosome translocation

$(2 ; 7)($ p11.2; 12.1$)$, suggesting a causal relationship between the rearrangement and the phenotype. FISH analysis using YAC and PAC clones as probes refined the breakpoint regions to genomic segments of about 164 and $120 \mathrm{~kb}$, respectively, providing a possible clue to pinpoint the location of a novel gene responsible for this rare muscle disorder.

European Journal of Human Genetics (2004) 12, 579-583. doi:10.1038/sj.ejhg.5201200

Published online 14 April 2004

Keywords: Brody disease; translocation; autosomal dominant; FISH; linkage

\section{Introduction}

Brody disease (BD [MIM 601003]) is an inherited, rare disorder of skeletal muscle function usually transmitted in an autosomal recessive fashion. The clinical presentation is characterized by exercise-induced progressive impairment of muscle relaxation, with stiffening, cramps and myalgias which may exacerbate in the cold. ${ }^{1}$ Symptoms usually resolve after few minutes' rest and can involve both upper and lower limbs and facial muscles, such as the eyelids. This clinical picture has often been described as 'pseudo-

*Correspondence: Dr EM Valente, CSS Mendel Institute, viale Regina Margherita 261, I-00198 Rome, Italy. Tel: + 39064416 0537; Fax: + 3906 4416 0548; E-mail: e.valente@css-mendel.it

Received 4 December 2003; revised 27 January 2004; accepted 4 March 2004 myotonia' or 'silent myotonia', as no myotonic discharges are recorded from muscles and contractures are electrically silent. Serum creatine kinase (CK) can be elevated and episodes of rhabdomyolysis and myoglobinuria may occur. Muscle biopsy morphology shows moderate degree of type 2 fibre atrophy and excess of internal nuclei. ${ }^{2}$ Biochemical and immunocytochemical studies in muscle biopsies from some patients with BD showed a marked reduction of $\mathrm{Ca}^{2+}$ uptake and $\mathrm{Ca}^{2+}$ ATPase activity in the sarcoplasmic reticulum of fast-twitch but not slow-twitch skeletal muscle fibres. ${ }^{2,3}$ This was followed by the identification of pathogenic mutations in the ATP $2 A 1$ gene, encoding SERCA1, the fast-twitch skeletal muscle sarcoplasmic reticulum $\mathrm{Ca}^{2+}$ ATPase, in a subset of patients with autosomal recessive BD. ${ }^{4}$ Rare families with autosomal dominant inheritance have been reported, but in those 
families mutations in the ATP2A1 gene could not be found, further supporting genetic heterogeneity. ${ }^{5-7}$ Here, we describe a novel Italian family with autosomal dominant BD and the physical mapping of the breakpoints of a balanced translocation $(2 ; 7)(\mathrm{p} 11.2 ; \mathrm{p} 12.1)$ cosegregating throughout three generations with the disease.

\section{Patients and methods Case report}

Family tree is shown in Figure 1. The proband (III:1) is a 30year-old man who came to our attention with a diagnosis of paramyotonia congenita (PMC). He had suffered since early childhood of exercise-induced episodes of painless muscle stiffness involving all muscular groups, the lower limbs being more severely affected. Muscle exercise is the only precipitating factor, and symptoms worsen with repeated exertion and exacerbate in cold weather. He also complains of occasional painful cramps in the dorsal and pectoral muscles; these cramps can occur at rest and usually resolve spontaneously within a few minutes. Episodes of periodic paralysis never occurred, however local myalgia and weakness usually follow severe episodes of muscle stiffening, lasting for a few hours. Warm-up

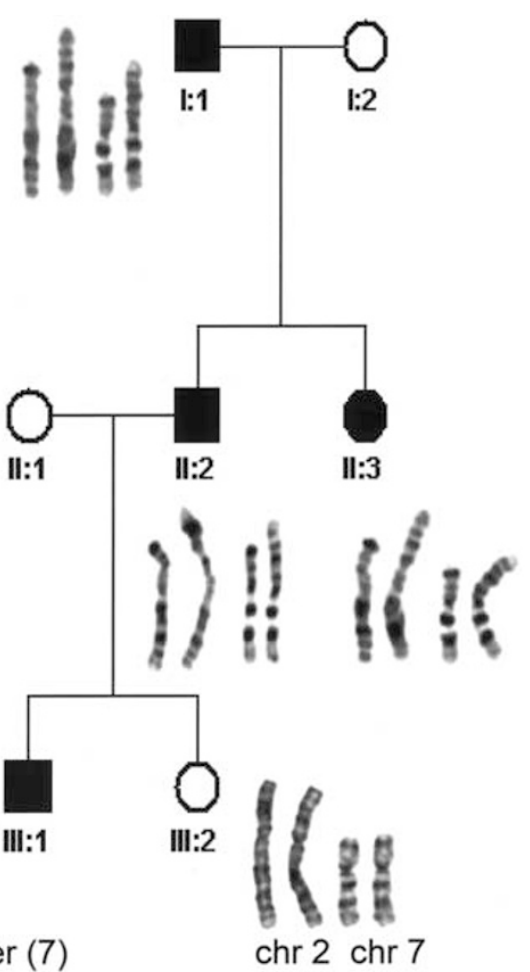

Figure 1 Pedigree of the family. Black symbols denote affected individuals. Standard cytogenetic analysis shows that all affected members are carries of $t(2 ; 7)(p 11.2 ; p 12.1)$, while the unaffected sister of the proband (III:2) carries a normal karyotype. phenomenon, transitory weakness, and 'second wind' phenomenon $^{8}$ did never occur. At the age of 21 years, following succinilcholine administration during an anaesthesiological procedure, the patient presented an episode of generalised muscle contracture associated with rhabdomyolysis and myoglobinuria.

Serum potassium, calcium and sodium were always in the normal range. Serum CK were constantly high (range 700-1300 U/1). Repeated electromyographic (EMG) examinations during episodes of stiffness showed that muscle contractures were always electrically silent. Although many muscles were tested, neither myotonic discharges nor other spontaneous activity could be detected. Mild myopathic changes were registered mainly from proximal muscles. A McManis long exercise test and a cold immersion test to $20^{\circ} \mathrm{C}$ did not reveal pathological decrements of compound muscle action potential, as it is often seen in muscle channelopathies. ${ }^{9}$ However, the long exercise test evoked a painless, electrically silent contracture of the abductor digiti minimi. Motor and sensory nerve conduction velocities were normal. At the age of 17 and 21 years, two muscle biopsies were performed, showing prevalence of type 2 fibres, of which $\sim 50 \%$ were atrophic, occasional central cores and increased number of central nuclei. Type 1 fibres were normal. An in vitro contracture test was positive to both halothane and caffeine, confirming the clinical suspect of malignant hyperthermia susceptibility (MHS). The sarcoplasmic reticulum $\mathrm{Ca}^{2+}$ uptake and SERCA1 activity were not measured. Neurological examination was unremarkable, except for the occasional cramps exerted with exercise. Muscular hypertrophy or weakness, percussion myotonia and myoedema were not observed. Verapamil (40 mg q.i.d.) provided some improvement in muscle relaxation, but it was discontinued after only 1 week as the patient was concerned about possible side effects.

The proband's father (II:2), aged 59 years, suffers from a milder clinical picture, with onset in late childhood and progressive worsening over the years. In his 20s, he had to give up his job as a shorthand-typist due to recurrent stiffness of his fingers, but he was able to run for some distance without problems. Now, he can only walk slowly and for very short distances, as exercise quickly exerts severe contractures of lower limb muscles. He never underwent anaesthesiological procedures. CK levels were mildly elevated (range 300-400 U/1). An EMG examination was normal, and no myotonic discharges were detected at rest or from stiffened muscles after exercise. A muscle biopsy, performed at the age of 49 years, showed variation in fibres size, moderate type 2 fibre atrophy and presence of cores and minicores. The in vitro contracture test was positive to caffeine only.

The proband's paternal aunt (II:3) and paternal grandfather (I:1) complained the same symptoms of exertional muscle stiffness, but to a lesser degree than the proband. 
They were not available for clinical examination but agreed to send a blood sample for molecular and cytogenetic studies. The only sister of the proband (III:2) is a 26-yearold lady who has never complained of any muscular symptom.

\section{Linkage analysis}

Genotyping was performed in all family members for microsatellite markers spanning the regions containing the following genes: ATP2A1 on chromosome 16p (D16S298$245 \mathrm{~kb}-A T P 2 A 1-1.7 \mathrm{Mb}-D 16 S 685) ; R Y R 1$ on chromosome 19q (D19S421-49kb-RYR1-92 kb-D19S422); SCN4A on chromosome $17 \mathrm{q}$ (D17S1154-1.0 Mb-SCN4A-758 kbD17S1809). The RYR1 locus was tested in light of the susceptibility to malignant hyperthermia demonstrated in at least two affected family members, while the SCN4A locus was analysed in order to definitely rule out the previous diagnosis of PMC.

Markers order and physical distances were obtained from the Human Genome Working Draft at http://genome.ucsc.edu. Genomic DNA fragments were PCR-amplified using fluorescent labelled primers, resolved on an ABI Prism 3100 DNA sequencer (Applied Biosystems, Foster City, CA, USA) and analysed with Genotyper software. Two-point linkage analysis was performed using the FASTLINK option of MLINK, assuming reduced penetrance (0.80), a disease gene frequency of 0.0001 and equal allele frequencies for each marker. ${ }^{10}$

\section{Standard cytogenetic and FISH analysis}

Chromosome analysis was performed in all family members according to routine GTG-banding techniques on cultured peripheral lymphocytes. YAC and BAC mapping data were obtained at http://www.ncbi.nlm.nih.gov/genome/clone/ (Figure 2a). Extracted DNA was biotin-16dUTP-labelled by Nick Translation kit (Invitrogen, Life Technologies, Carlsvad, CA, USA). FISH analysis was carried out according to previously published methods. ${ }^{11}$ Painting FISH was performed using chromosomes 2 and 7 painting probes (Vysis, Inc., Downers Grove, IL, USA) according to the producer protocol.

\section{Results}

Negative pairwise LOD scores were obtained for all markers spanning the ATP2A1, RYR1 and SCN4A intervals (Table 1), allowing the exclusion of these three loci as responsible for the disease in this family. Calculation of pairwise LOD scores assuming different penetrance values (0.40-1.00) did not result in a significant change.

Standard cytogenetic analysis showed a balanced translocation (2p11.2;7p12.1) karyotype in all affected individuals, while the karyotype was normal in the healthy sister of the proband (Figure 1). FISH painting of chromosomes 2 and 7 excluded that other chromosomes were involved in the rearrangement.

In order to refine the translocation breakpoints, a series of FISH with YACs and BACs mapping on chromosome $2 \mathrm{p}$ and 7p was carried out (Figure 2a). The RP11-269K22 BAC clone on chromosomes 2 was split, the fluorescent signal being detected on both the derivative chromosomes (Figure 2b). The breakpoint region was therefore restricted to $\sim 164 \mathrm{~kb}$ in $2 \mathrm{p} 11.2$. On chromosome 7 , the RP11175C14 BAC clone was split (Figure 2c) defining the translocation breakpoint on chromosome 7 p12.1 within a $\sim 120 \mathrm{~kb}$ fragment.

\section{Discussion}

Autosomal dominant BD is a very rare condition and only few families have been reported in literature. ${ }^{3,5-7}$ The phenotype of the four affected individuals in the present family and routine histochemical studies of skeletal muscle are consistent with the diagnosis of $\mathrm{BD}$, although microsomal $\mathrm{Ca}^{2+}$ uptake and activity of SERCA1 in bioptic muscle were not measured.

In the absence of biochemical or immunocytochemical data, other differential diagnoses must be considered. The index case came to our attention with a diagnosis of PMC (MIM [168300]), which is characterised by cold-induced stiffness and weakness, with myotonia worsening with progressive exercise (paradoxical myotonia). However, unlike PMC and all other myotonic disorders, muscular contractures were always silent and no clinical or electrical myotonia could be demonstrated. Involvement of the SCN4A gene, which is responsible for PMC and hyperkalaemic periodic paralysis (HyPP), was ruled out by linkage analysis (Table 1 ).

An in vitro contracture test both in the proband and his father was positive for MHS (MIM [145600]). This condition can occur alone or in conjunction with other muscle disorders, such as central core disease, HyPP and also BD. In fact, a contracture test was found positive in one patient with biochemically proven deficit of SERCA $1 .^{2}$ MHS can be inherited in an autosomal dominant fashion and is genetically heterogeneous, with more than half of the families harbouring mutations in the RYR1 gene. Linkage to RYR1 was also excluded (Table 1 ).

In the present family, a balanced chromosome translocation cosegregates with autosomal dominant BD, suggesting a causal relationship between this rearrangement and the disease. FISH analysis allowed a close definition of the breakpoints. The two interrupted clones span 164 and $120 \mathrm{~kb}$ on chromosomes 2p11.2 and 7p12.1, respectively. These intervals might contain possible candidate genes, as a submicroscopic deletion or an incidental gene rupture could have occurred during the translocation event. Two genes map to the BAC containing chromosome 2 break- 
a

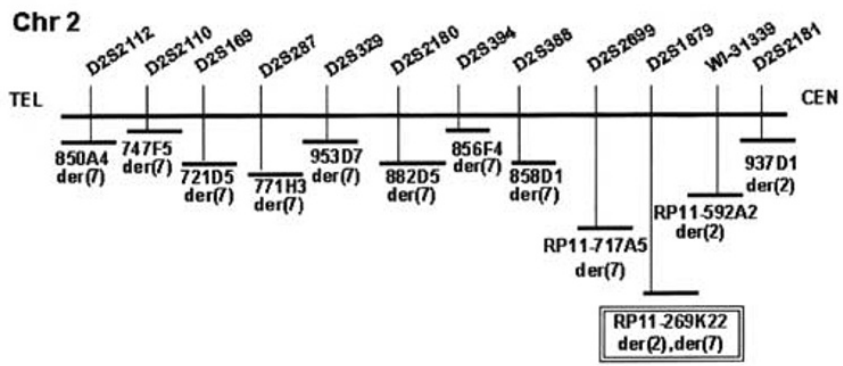

Chr 7

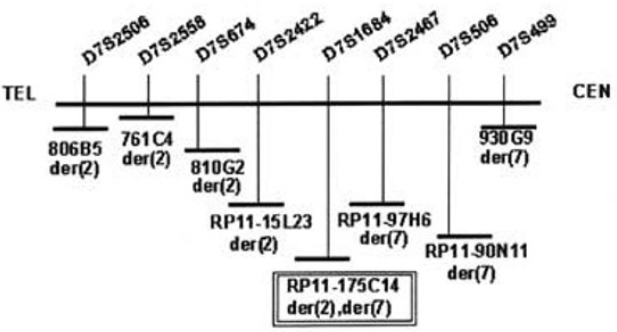

Markers

YACs

BACs

\section{Markers}

YACs

BACs

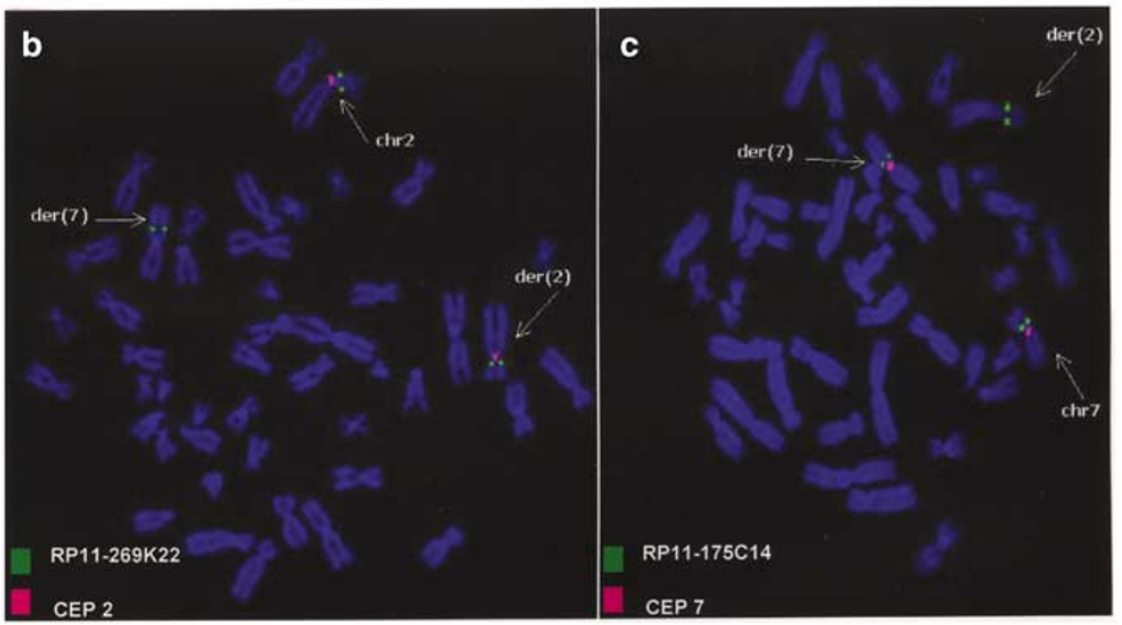

Figure 2 FISH analysis of the $(2 ; 7)$ translocation breakpoints. (a) Map of YAC and BAC probes and relative markers used for FISH analysis to refine the breakpoint regions on chromosomes 2 and 7 . Boxed clones contain the translocation breakpoints. (b) RP11-269K22, mapping to 2p11.2, shows hybridisation signal on both derivative chromosomes and on normal chromosome 2. (c) RP11-175C14, mapping to 7p12.1, shows hybridisation signal on both $\operatorname{der}(7)$ and der(2), and on normal chromosome 7 .

point, RNF103 encoding a ring-finger protein and NEDF, encoding the neuroendocrine differentiation factor. Although the function of these genes is still partly uncharacterised, none of them appears to be a strong candidate for BD. The BAC sequence including chromosome 7 breakpoint contains only two unspliced ESTs (AI218435 and AI902491). It is yet unclear if these are translated into functional proteins.

Alternatively, a position effect may be considered. A change of gene expression due to a chromosomal aberration disrupting regulatory sequences but not the transcrip- tional unit has been reported for several disorders. ${ }^{12} \mathrm{~A}$ bioinformatic analysis of the neighbouring regions of the two breakpoints identified several putative candidate genes. On chromosome 2, two genes appeared particularly intriguing. IMMT, distant $\sim 400 \mathrm{~kb}$ from the interrupted $\mathrm{BAC}$, encodes a mitochondrial protein (mitofilin) mainly expressed in muscle, which acts as an ATP-driven motor protein interacting with cytoskeletal components. ${ }^{13}$ The VAMP5 gene, distant $\sim 1 \mathrm{Mb}$ from the breakpoint, encodes myobrevin, a member of the vesicle-associated membrane protein (VAMP)/synaptobrevin family preferentially 
Table 1 Pairwise LOD scores between BD and markers spanning the ATP2A1, RYR1 and SCN4A loci on chromosomes 16p, $19 q$ and $17 q$

\begin{tabular}{lccccrr}
\hline & \multicolumn{7}{c}{ LOD scores at $\theta=$} \\
\cline { 2 - 7 } Markers & 0.0 & 0.01 & 0.05 & 0.1 & 0.2 & 0.3 \\
\hline D16S298 & -4.27 & -1.86 & -1.14 & -0.81 & -0.49 & -0.29 \\
D16S685 & -3.88 & -2.15 & -1.40 & -1.03 & -0.62 & -0.36 \\
D17S1154 & -3.80 & -1.56 & -0.84 & -0.52 & -0.21 & -0.06 \\
D17S1809 & -3.80 & -1.56 & -0.84 & -0.52 & -0.21 & -0.14 \\
D19S421 & -4.27 & -2.86 & -1.52 & -0.96 & -0.45 & -0.19 \\
D19S422 & -4.27 & -2.86 & -1.52 & -0.96 & -0.45 & -0.19 \\
\hline
\end{tabular}

expressed in skeletal muscle, and likely involved in membrane vesicles trafficking. ${ }^{14}$ A very interesting gene, FKBP9, is located at $\sim 3 \mathrm{Mb}$ from the interrupted BAC on chromosome 7 . This gene is highly expressed in skeletal muscle and encodes the FK506 binding protein 9, which contains two EF-hand calcium-binding domains. FKBP9 bears close similarities with the cardiac muscle protein FKBP1B, which binds to the cardiac ryanodine receptor RYR2 and modulates its activity. ${ }^{15}$ The ryanodine receptors are calcium channels located in the sarcoplasmic reticulum of skeletal (RYR1) and cardiac (RYR2) muscle, and play an essential role in intracellular calcium signalling and muscle contraction. In this light, other genes involved in the same calcium regulation process represent excellent candidates for BD. However, it is still not possible to foresee how distantly a position effect can act. ${ }^{16,17}$

In conclusion, we describe a novel family with autosomal dominant Brody disease bearing a $(2 ; 7)$ chromosomal translocation. Linkage analysis in other autosomal dominant $\mathrm{BD}$ families with markers spanning the rearranged genomic regions, along with sequencing of critical regions and long-range expression studies of the candidate genes, could be decisive to map a novel $\mathrm{BD}$ locus and ease the identification of the responsible gene.

\section{Acknowledgements}

We are gratefully indebted to all family members and treating physicians, in particular Dr Luigi Bettoni, for their kind cooperation. We thank Dr Cinzia Felicita Sala (YAC Screening Center HSR-DIBIT, Milan, Italy) and Dr Mariano Rocchi (Resources for Molecular Cytogenetics, Bari, Italy) for the generous gift of YAC, BAC and PAC clones. We also acknowledge the financial support of the Italian Ministry of Health (Ricerca Finalizzata; Ricerca Corrente).

\section{References}

1 Brody TA: Muscle contracture induced by exercise. A syndrome attributable to decreased relaxing factor. N Engl J Med 1969; 290: $187-192$

2 Karpati G, Charuk J, Carpenter S, Jablecki C, Holland P: Myopathy caused by a deficiency of $\mathrm{Ca}^{2+}$-adenosine triphosphatase in sarcoplasmic reticulum (Brody's disease). Ann Neurol 1986; 20: 38-49.
3 Benders AAGM, Veerkamp JH, Oosterhof A et al: $\mathrm{Ca}^{2+}$ homeostasis in Brody's disease. A study in skeletal msucle and cultured muscle cells and the effects on Dantrolene and Verapamil. J Clin Invest 1994; 94: 741-748.

4 Odermatt A, Taschner PE, Khanna VK et al: Mutations in the gene-encoding SERCA1, the fast-twitch skeletal muscle sarcoplasmic reticulum $\mathrm{Ca}^{2+}$-ATPase, are associated with Brody disease. Nat Genet 1996; 14: 191-194.

5 Danon MJ, Karpati G, Charuk J, Holland P: Sarcoplasmic reticulum adenosine triphosphatase deficiency with probable autosomal dominant inheritance. Neurology 1988; 38: $812-815$.

6 Zhang Y, Fujii J, Phillips MS et al: Characterization of cDNA and genomic DNA encoding SERCA1, the $\mathrm{Ca}^{2+}$-ATPase of human fasttwitch skeletal muscle sarcoplasmic reticulum, and its elimination as a candidate gene for Brody disease in three patients. Genomics 1995; 30: 415-424.

7 Odermatt A, Taschner PE, Scherer SW et al: Characterization of the gene encoding human sarcolipin (SLN), a proteolipid associated with SERCA1: absence of structural mutations in five patients with Brody disease. Genomics 1997; 45: $541-553$.

8 Braakhekke JP, de Bruin MI, Stegeman DF, Wevers RA, Binkhorst RA, Joosten EMG: The second wind phenomenon in McArdle's disease. Brain 1986; 109: 1087-1101.

9 McManis PG, Lambert EH, Daube JR: The exercise test in periodic paralysis. Muscle Nerve 1986; 9: 704-710.

10 Cottingham Jr RW, Idury RM, Schaffer AA: Faster sequential genetic linkage computations. Am J Hum Genet 1993; 53: $252-263$.

11 Chong SS, Pack SD, Roschke AV et al: A revision of the lissencephaly and Miller-Dieker syndrome critical regions in chromosome 17p13.3. Hum Mol Genet 1997; 6: 147-155.

12 Kleinjan D-J, van Heyningen V: Position effect in human genetic disease. Hum Mol Genet 1998; 7: 1611-1618.

13 Icho T, Ikeda T, Matsumoto Y, Hanaoka F, Kaji K, Tsuchida N: A novel human gene that is preferentially transcribed in heart muscle. Gene 1994; 144: 301-306.

14 Zeng Q, Subramaniam VN, Wong SH et al: A novel synaptobrevin/VAMP homologous protein (VAMP5) is increased during in vitro myogenesis and present in the plasma membrane. Mol Biol Cell 1998; 9: 2423-2437.

15 Masumiya H, Wang R, Zhang J, Xiao B, Chen SR: Localization of the 12.6-kDa FK506-binding protein (FKBP12.6) binding site to the NH2-terminal domain of the cardiac $\mathrm{Ca}^{2+}$ release channel (ryanodine receptor). J Biol Chem 2003; 278: 3786-3792.

16 Wirth J, Wagner T, Meyer J et al: Translocation breakpoints in three patients with campomelic dysplasia and autosomal sex reversal map more than $130 \mathrm{~kb}$ from SOX9. Hum Genet 1996; 97: 186-193.

17 Lettice L, Horikoshi T, Heaney SJH et al: Disruption of a longrange cis-acting regulator for Shh causes preaxial polydactyly. Proc Natl Acad Sci USA 2002; 99: 7548-7553. 